\title{
Magnetic-Field-Dependent Energy Levels in a Highly Anisotropic Electronic Material
}

\author{
A. S. Perel, ${ }^{(1),(2)}$ J. S. Brooks, ${ }^{(1),(2)}$ C. J. G. N. Langerak, (3),(a) T. J. B. M. Janssen, (3) J. Singleton, ${ }^{(3),(b)}$ \\ J. A. A. J. Perenboom, ${ }^{(3)}$ and L. Y. Chiang ${ }^{(4)}$ \\ ${ }^{(1)}$ Department of Physics, Boston University, Boston, Massachusetts 02215 \\ ${ }^{(2)}$ F. B. National Magnet Laboratory, Massachusetts Institute of Technology, 170 Albany Street, \\ Cambridge, Massachusetts 02139 \\ ${ }^{(3)}$ High Field Magnet Laboratory, University of Nijmegen, Nijmegen, The Netherlands \\ ${ }^{(4)}$ EXXON Research and Engineering Company, Annandale, New Jersey 08801
}

(Received 3 May 1991)

\begin{abstract}
We report the direct observation of magnetic-field-dependent energy levels in an anisotropic twodimensional electron gas. The experiment was carried out by far-infrared-reflectivity measurements on a well studied member of the Bechgaard salts, (TMTSF) ${ }_{2} \mathrm{ClO}_{4}$, at low temperatures. We find two distinct bands above a threshold field and a number of unusual features which we interpret in terms of the underlying quantized field-induced spin-density-wave subphases. We also observe in the optical data details associated with anomalies in this material in high magnetic fields.
\end{abstract}

PACS numbers: $74.70 . \mathrm{Kn}, 73.20 . \mathrm{Dx}, 75.30 . \mathrm{Fv}$

For the case of a quasi-one-dimensional (highly anisotropic) electron gas it has been shown theoretically [1] that, in the presence of a magnetic field, the system is unstable against the formation of an anisotropic twodimensional electron gas (A2DEG). The theoretical energy spectrum for the A2DEG has been recently determined [2] and several distinct features are introduced by the anisotropy: (1) There is a spin-density-wave (SDW) gap at the Fermi level, and a spectrum of Landau bands, both above and below the SDW gap; (2) the quantized SDW nesting vector (with quantum number $N$ ) is magnetic-field dependent, and both the SDW gap and the Landau band scheme change at each new value of $N$, with the most dramatic change occurring at the $N=1$ to $N=0$ transition where many gaps vanish.

The Bechgaard salts, and in particular (TMTSF) ${ }_{2} \mathrm{ClO}_{4}$ and (TMTSF) ${ }_{2} \mathrm{PF}_{6}$, are physical representations of quasi-one-dimensional electronic materials [3,4]. At a threshold magnetic field $\boldsymbol{H}_{\text {th }}$ applied along the least conducting direction $c^{*}$, these materials exhibit quantized SDW behavior [3] and an integer quantum Hall behavior in the $a^{*}-b^{*}$ plane $[5,6]$. Specific-heat measurements on (TMTSF) ${ }_{2} \mathrm{ClO}_{4}$ have given thermodynamic evidence for the main SDW gap, but deviations from a simple weakcoupling picture arise [7]. In (TMTSF) ${ }_{2} \mathrm{ClO}_{4}$, a number of anomalies become apparent in transport [8,9], magnetization [10], specific-heat [11], and thermopower [12] measurements. The two main anomalies are the Shubnikov-de Haas- $(\mathrm{SdH}-)$ like rapid oscillations which coexist with the SDW phases, and the reentrance from a SDW to a metallic phase at high fields. We note that many of the unusual features of this material are highly dependent on the ordering of the tetrahedral anions at the 24-K transition. The high-field behavior recently has been the subject of controversy and considerable theoretical attention [13-15].

We have undertaken far-infrared-(FIR-) reflectivity measurements [16] on (TMTSF) ${ }_{2} \mathrm{ClO}_{4}$ to directly deter- mine the magnetic-field-dependent energy spectrum of the A2DEG and to explore optically the very-highmagnetic-field anomalies. A parquet of a dozen single crystals of (TMTSF) ${ }_{2} \mathrm{ClO}_{4}$ were arranged with their $c$ axes parallel to the applied magnetic field. Transport leads were attached to one sample placed outside the range of the light, which was unpolarized in this investigation. Reflectance measurements were made with a chopped FIR laser (approximately twenty different laser energies in the range 1-20 meV were used) and a light pipe which ended in a cone with a 1 -mm-diam hole $2 \mathrm{~mm}$ above the parquet. Incident and reflected radiation signals were detected bolometrically with thin slices from a single Allen-Bradley resistor. Phase-sensitive detection at a chopper frequency near $30 \mathrm{~Hz}$ was used, and the ratio of the reflected and incident signals was recorded as a function of magnetic field for fixed temperature and photon energy. The samples were slow cooled through the anion ordering transition at $24 \mathrm{~K}$, and several magnet and cryogenic arrangements were employed in the measurements.

The main results of this work are summarized in Figs. 1 and 2. The normalized reflectivity change $\Delta R / R_{0}$ versus magnetic field and photon energy for five field sweeps at a temperature of $1.2-\mathrm{K}$ run is shown in Fig. 1 and in Fig. 2 we have plotted energy- and magneticfield-dependent structure in $\Delta R / R_{0}$. Our measurements address three different ranges in the phase diagram (determined from previous experiments [7-12]) indicated in Fig. 2.

(a) The vicinity of the threshold field and multiple subphase region is shown in Fig. 1. Here several features are immediately evident. First, there is a pronounced change in the base line of $\Delta R / R_{0}$ above the threshold field $H_{\mathrm{th}}$, the sign of which is energy dependent. The resonant character of this feature is evident in the inset of Fig. 1 where the fractional change in reflectivity at the metalto-SDW phase transition is shown. Second, there are 


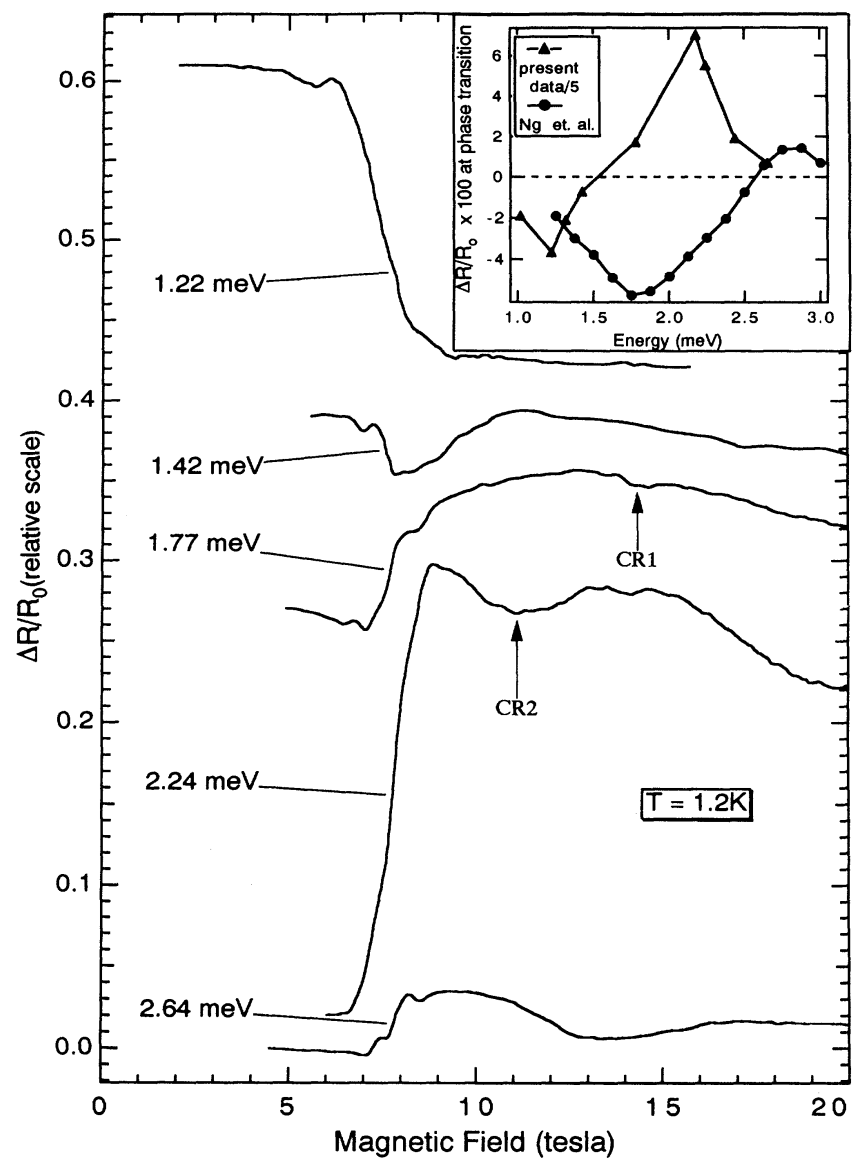

FIG. 1. Relative change in reflectivity $\Delta R / R_{0}$ vs magnetic field at $1.2 \mathrm{~K}$ for different laser energies. The traces are offset for clarity. Inset: The change in reflectivity (SDW signal subtracted from the metallic sample) vs energy for field-induced (triangles) and quench-induced (dots) SDW phases. The relative values of the present data have been reduced by a factor of 5 for comparison with the previous absolute reflectance results.

magnetic-field-dependent features (dips) characteristic of cyclotron-resonance (CR) behavior. And third, significant additional structure appears above $\boldsymbol{H}_{\text {th }}$.

(b) At intermediate fields in the $N=0$ SDW phase, we show in Fig. 3(a) the appearance of structure with the periodicity of the $\mathrm{SdH}$ rapid-oscillation magnetoresistance signal. In Fig. 3(b) we observe both CR2 and the oscillations fields above CR2.

(c) At the highest magnetic fields we explored the high-field reentrant phase boundary, as shown in Figs. $3(c)$ and 3(d). The magnetoresistance of the transport sample indicates the position of the reentrant phase line. At the lowest temperature, $\Delta R / R_{0}$ shows a dip at the reentrance, the nature of which changes at the highest photon energy. At intermediate temperatures, only one energy was possible, and a very broad feature was identified with a change of slope in the reentrant region. At the highest temperature, a sharp drop at reentrance
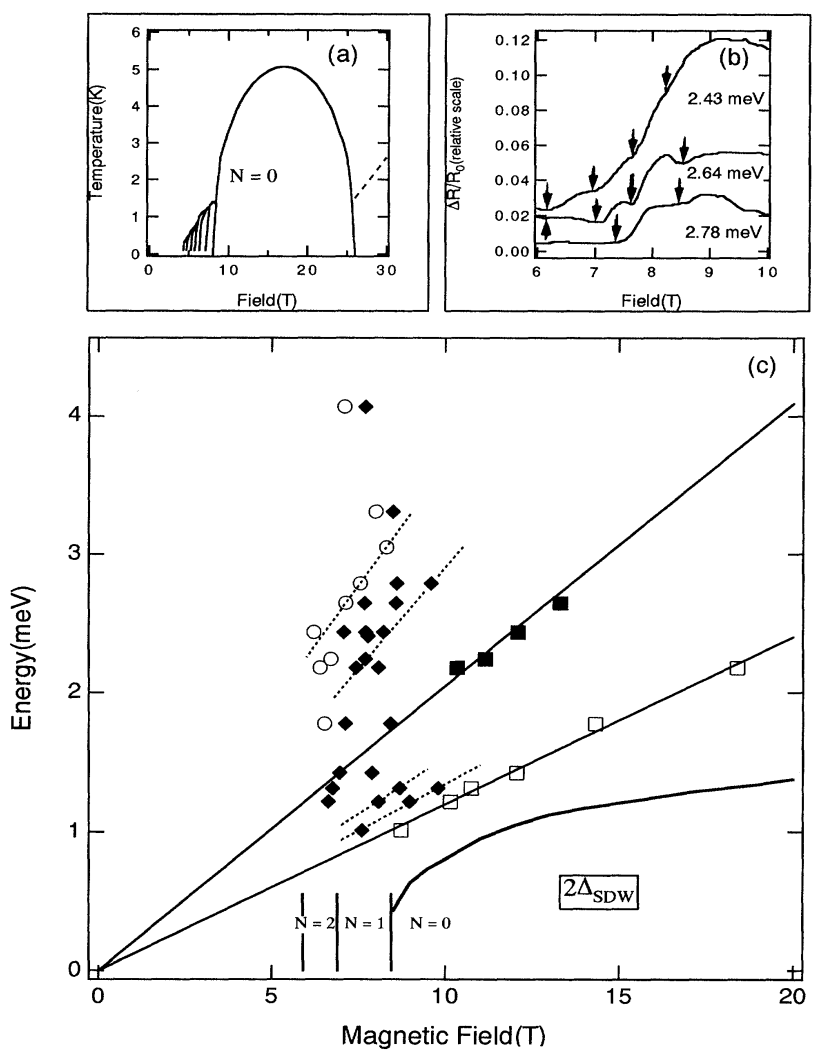

FIG. 2. (a) Phase diagram of (TMTSF) ${ }_{2} \mathrm{ClO}_{4}$. (b) Detail of energy-dependent structure in $\Delta R / R_{0}$ above $H_{\text {th }}$ for representative photon energies. Arrows indicate features used in (c). (c) Energy- and magnetic-field dependent features of the reflectivity signal at $1.2 \mathrm{~K}$. Open squares, $\mathrm{CR} 1$; large closed squares, CR2; small closed squares, position of additional features; open circles, position of onset of change in $\Delta R / R_{0}$; dotted lines, energy-dependent structure.

was observed for all three energies investigated.

An important feature of the present work is that a SDW state can be induced with magnetic field. Hence the energy-dependent difference between the metallicand SDW-state optical signals are easily observed, as indicated in the inset of Fig. 1. To compare these results with previous interferometry work, we have taken the difference between the reflectance on relaxed (metallic) samples [17] and the reflectance on quenched (SDW) samples [18]. This difference, which is for the electric field polarized along the $a^{*}$ axis of the sample at zero magnetic field, is also presented in the inset of Fig. 1. There is some similarity in the resonant character, but it is difficult to make a quantitative comparison between the two types of measurements. The SDW state in quenched samples may be highly sample dependent [19], uncertainties due to diffraction effects [20] and mode switching may come into play, and polarization may play a role. Nevertheless, the energy-dependent nature of the background signal (in our results for instance) reverses sign 


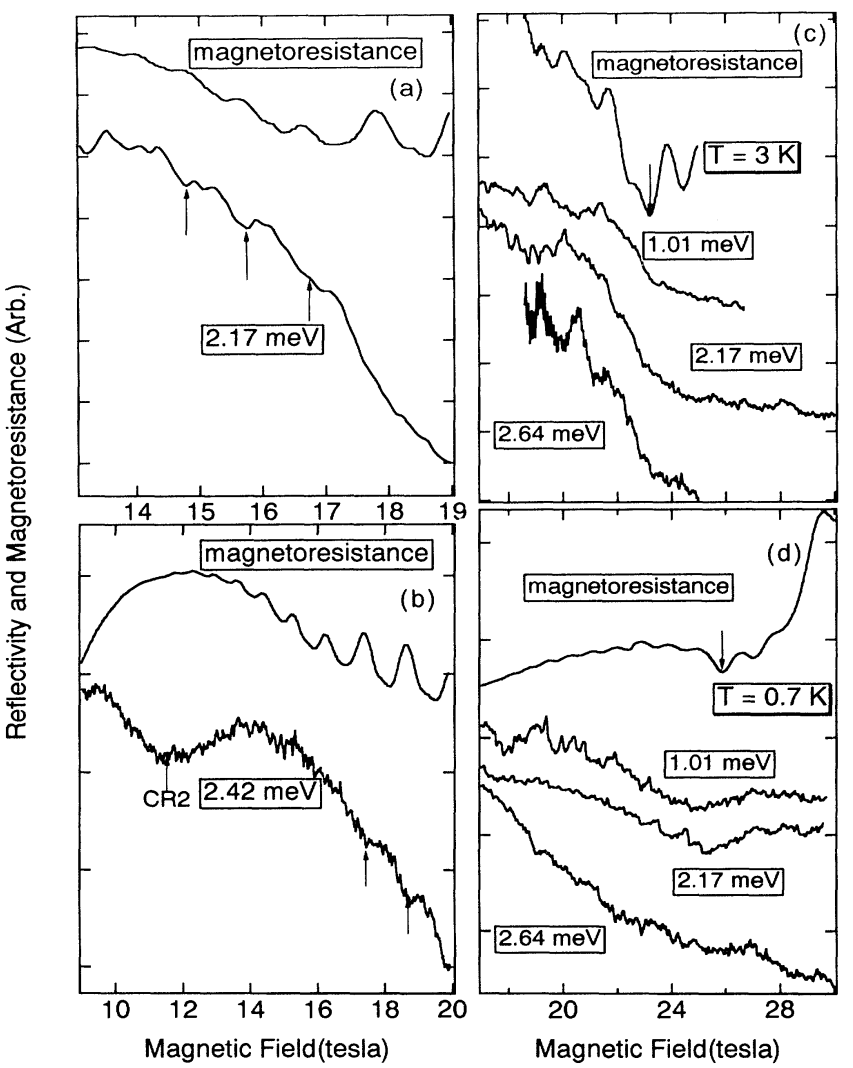

FIG. 3. Rapid-oscillation signals (magnetoresistance and reflectivity) in a highly ordered sample. Arrows indicate field of reentrance from magnetoresistance signal. (a) Over a small range of field at $2.17 \mathrm{meV}$. (b) Over a larger range of field at $2.42 \mathrm{meV}$ where both $\mathrm{CR}$ and the oscillations are evident. (c),(d) Energy- and temperature-dependent investigation of the reentrant regime. Vertical axis units are arbitrary.

near $1.5 \mathrm{meV}(=17 \mathrm{~K})$. In the usual BCS mean-field treatment, where $2 \Delta / k_{B} T=3.5$, the $N=0$ SDW gap $2 \Delta_{\text {SDW }}$ is $16 \mathrm{~K}$ [see Fig. $2(\mathrm{a})$ ]. In light of previous work on quench-induced SDW (TMTSF) ${ }_{2} \mathrm{ClO}_{4}$ and the close correspondence of mean-field and optical energies, this effect seems highly correlated with the opening of the SDW gapped states above $H_{\text {th }}$.

Unlike the broad variations in background signal discussed above, the energy- and field-dependent features in the data summarized in Fig. 2 give a clearer account of the underlying gap spectrum. The density of states in the field-induced SDW states change at each subphase, hence some of the changes in reflectivity observed arise from passage from one SDW gap to another, and should correspond to the positions of the SDW phase boundaries and be independent of energy. However, much of the structure we observe is energy dependent in magnetic field. We attribute the energy-dependent structure which in Fig. 2 is most apparent between $H_{\text {th }}$ and the $N=0$ state as evidence for excitations across the SDW gap, as well as intraband transitions between the theoretically predict- ed Landau bands. Between the $N=1$ and $N=0$ phases, the most dramatic change (reduction) in the predicted band structure takes place, an effect which is also apparent in the data in Fig. 2.

In an attempt to begin unraveling the details of the spectrum, we have labeled the two prominent cyclotronresonant features $\mathrm{CR} 1$ and $\mathrm{CR} 2$, which by simple analysis yield effective masses of $m^{*} / m \approx 1.0(\mathrm{CR} 1)$ and $m^{*} / m \approx 0.6$ (CR 2 ), where $m$ is the free electron mass. CR2 in particular has a large linewidth which increases with magnetic field, as expected for an increasing Landau band width with magnetic field. CR1 is the lowestenergy line observed in our data, and is very close to the gap edge expected from the mean-field SDW gap value in Fig. 2. CR 1 may indeed represent the excitation across the SDW gap.

CR2 might also involve states above the gap, but it could have an entirely different origin. In Fig. 3 we show the rapid oscillations both in the transport and in the reflectivity, where in one case the field range is sufficient to see CR2 at a field below the oscillations. We note that for a 2DEG, Ando [21], first showed that SdH oscillations should be superimposed on the cyclotron-resonance signal, an effect which has been seen experimentally [16]. Other than a striking similarity between Fig. 3(b) and the Ando picture, we cannot with certainty make the association between CR2 and the rapid oscillations. Additional evidence lies in the consideration of the rapidoscillation frequency of $255 \mathrm{~T}$ [9] under the assumption of a circular $k$-space orbit. The period $\Delta(1 / B)$ of $\mathrm{SdH}$ oscillations is related to the effective mass and Fermi velocity $v_{F}$ by the relation $\Delta(1 / H)=2 e \hbar / m^{* 2} v_{F}^{2}$. The Fermi velocity is approximated [22] to be $2 \times 10^{5} \mathrm{~m} / \mathrm{s}$ which gives a rapid-oscillation effective mass of $m^{*} / m=0.5$, in very close agreement with the corresponding mass for CR2. In order to unambiguously assign one of the CR lines with the rapid-oscillation phenomena, a cyclotronresonance measurement is needed below $H_{\text {th }}$ where the SDW phases cease to exist but the rapid oscillations are observed. Although the metallic state, the low energy, and the anisotropy complicate the measurement, it should be possible.

We close our discussion of the results by considering the reentrant transition above the $N=0$ state. Here, at least at temperatures above $2 \mathrm{~K}$, the material returns to a metallic state. At low temperatures, there is mounting evidence that the state is gapped. Our high-temperature data show a sharp drop in $\Delta R / R_{0}$ at the transition, which may represent the return of metallic behavior observed below $\boldsymbol{H}_{\text {th }}$; our low-temperature data show a dip followed by a much less dramatic change: This may represent a transition from the $N=0$ state to another gapped state at higher fields, with an intervening metallic phase at the transition. This interpretation is consistent with both specific-heat [11] and thermopower [12] measurements above the reentrant phase line.

In summary, we have used far-infrared-reflectivity 
measurements to determine the energy spectrum of an anisotropic electronic material in high magnetic fields. This is the first such determination of the magnetic-fieldinduced energy levels in an organic conductor. Our results fit well with the theoretical expectations of a fieldinduced spin-density-wave gap at the Fermi level and with a hierarchy of Landau bands. We argue that the two main resonant lines $\mathrm{CR} 1$ and $\mathrm{CR} 2$ are associated with the SDW gap and rapid-oscillation phenomena, respectively. Further, detailed interferometry work in high magnetic fields will be necessary to unravel the details of the energy spectrum of the A2DEG system. Finally, we have explored the reentrant phase line and find consistency between optical and thermodynamic measurements.

This work was supported by NSF-DMR 88-18510 and NATO 0335/88. We are grateful to the staff of the Nijmegen High Field Magnet Laboratory (supported by the Dutch FOM) for their very high level of support in carrying out this work, and to Paul Chaikin's group at Princeton (supported by NSF-DMR 88-22532) for providing the samples used. We further acknowledge useful discussions with Gilles Montambaux and Jan van Bentum.

(a) Present address: Department of Physics, University of Nottingham, Nottingham NG7 2RD, United Kingdom.

(b) Present address: Clarendon Laboratory, Oxford University, Oxford OX1 3PU, United Kingdom.

[1] L. P. Gor'kov and A. G. Lebed, J. Phys. (Paris), Lett. 45, L433 (1984); P. M. Chaikin, Phys. Rev. B 31, 4770 (1985); G. Montambaux, M. Heritier, and P. Lederer, J. Phys. (Paris), Lett. 45, L533 (1984).

[2] G. Montambaux and D. Poilblanc, Phys. Rev. B 37, 1913 (1988).

[3] See, for instance, G. Montambaux, M. J. Naughton, R. V. Chamberlin, X. Yan, P. M. Chaikin, and M. Ya. Az- bel, Phys. Rev. B 39, 885 (1989).

[4] For a recent review of these materials, see T. Ishiguro and K. Yamaji, Organic Superconductors (Springer-Verlag, Berlin, 1990).

[5] S. T. Hannahs et al., Phys. Rev. Lett. 63, 1988 (1989).

[6] J. Cooper et al., Phys. Rev. Lett. 63, 1984 (1989).

[7] F. Pesty et al., Phys. Rev. Lett. 55, 969 (1985).

[8] R. V. Chamberlin et al., Phys. Rev. Lett. 60, 1189 (1988).

[9] X. Yan, M. J. Naughton, R. V. Chamberlin, S. Y. Hsu, L. Y. Chiang, J. S. Brooks, and P. M. Chaikin, Phys. Rev. B 36, 1799 (1987).

[10] M. J. Naughton et al., Phys. Rev. Lett. 61, 621 (1988).

[11] N. A. Fortune et al., Phys. Rev. Lett. 64, 2054 (1990).

[12] R. C. Yu et al., Phys. Rev. Lett. 65, 2458 (1990).

[13] V. M. Yakovenko, Zh. Eksp. Teor. Fiz. 93, 627 (1987) [Sov. Phys. JETP 66, 355 (1987)].

[14] A. G. Lebed and P. Bak, Phys. Rev. B 40, 11433 (1989).

[15] K. Machida and Y. Hori (to be published); A. G. Lebed, in Proceedings of the Eleventh General Conference of the Condensed Matter Division, Exeter, United Kingdom, 8-11 April 1991 [Phys. Scr. (to be published)].

[16] C. Langerak, Ph.D. thesis, University of Nijmegen (unpublished). Many of the details of the techniques used in our work, and representative results for FIR reflectivity studies on both bulk and 2DEG semiconductor materials, are given here.

[17] H. K. Ng, T. Timusk, and K. Bechgaard, J. Phys. (Paris), Colloq. 44, C3-867 (1983).

[18] H. K. Ng and T. Timusk, Mol. Cryst. Liq. Cryst. 119, 191 (1985).

[19] M. Takigawa and G. Saito, Physica (Amsterdam) 143B, 422 (1986).

[20] T. Timusk, in Low Dimensional Conductors and Superconductors, edited by D. Jerome and L. G. Caron (Plenum, New York, 1987); J. van Bentum (private communication).

[21] T. Ando, J. Phys. Soc. Jpn. 38, 989 (1975).

[22] R. V. Chamberlin et al., Phys. Rev. Lett. 61, 2277 (1988). 